\title{
A CHARACTERIZATION OF UNIFORM PARACOMPACTNESS
}

\author{
J. FRIED AND Z. FROLIK
}

\begin{abstract}
Main result: a uniform space $X$ is uniformly paracompact [R] iff for some (and then any) compactification $K$ of $X$ and for any compact $C \subset K \backslash X$ closed disjoint sets $X \times C$ and the diagonal $\Delta_{X}(=\{\langle x, x\rangle \mid x \in X\})$ can be separated by a uniformly continuous function on the semiuniform product $X * K$.
\end{abstract}

H. Tamano gave [T] the following interesting result. For a $T_{1}$-completely regular space $X$ the following conditions are equivalent:

(a) $X$ is paracompact,

(b) $X \times \beta X$ is normal, i.e. disjoint closed sets can be separated by a continuous function,

(c) for each compact $C \subset \beta X \backslash X$ the two closed sets $X \times C$ and the diagonal $\Delta_{X}=\{\langle x, x\rangle \mid x \in X\}$ can be separated by a continuous function on $X \times \beta X$.

There are several ways to generalize this result to uniform spaces. Separation of closed sets in the usual (i.e. categorical) product by various types of functions has been considered by $Z$. Frolik in $\left[F_{2}\right]$; in this case $(b)$ is always equivalent to (c). The same author studied separation of closed sets by coz-functions if the product is interpreted as the semiuniform product $*$ (in the sense of Isbell [ $\mathbf{I}_{1}, \mathbf{I I I}$, p. 44]), (b) and (c) are not equivalent in this situation, indeed, they give characterizations of two distinct classes of uniform spaces [ $\left.\mathbf{F}_{3}\right]$.

Here we study the case when the product is * and separating functions are uniformly continuous. Since the properties studied do not depend on the compactification we use (by a compactification of a uniform space we mean a compactification of the underlying topological space), the Samuel compactification is just one of compactifications. Definition of $*$ and properties we need are recalled in $\S 1$.

If not stated otherwise, by a space we mean uniform $T_{2}$-space. Following M. D. Rice [R], a space $X$ is called uniformly paracompact if every open cover of $X$ has a uniformly locally finite open refinement. Again, we recall the necessary facts in $\$ 1$, more detailed information can be found in $\left[\mathbf{H}_{1}\right]$.

The reader is referred to Isbell's book $\left[\mathbf{I}_{1}\right]$ for definitions and basic facts concerning uniform spaces.

We can now formulate our results.

THEOREM 1. A space $X$ is uniformly paracompact if and only if for some, and then any, compactification $K$ of $X$ the following holds:

Received by the editors January 10, 1983.

1980 Mathematics Subject Classification. Primary 54E15, 54D18.

(1)1983 American Mathematical Society $0002-9939 / 83 \$ 1.00+\$ .25$ per page 
for each compact $C \subset K \backslash X$ there exists a uniformly continuous function $f$ on $X * K$ such that $f$ is 1 on $\Delta_{X}$ and $f$ is 0 on $X \times C$.

THEOREM 2. A space $X$ is fine and paracompact if and only if for some, and then any, compactification $K$ of $X$ the following holds:

for each pair $F_{0}, F_{1}$ of closed disjoint sets on $X \times K$ there exists a uniformly continuous function $f$ on $X * K$ such that $f$ is 0 on $F_{0}$, and $f$ is 1 on $F_{1}$.

COROLLARY. The following conditions are equivalent:

(1) $X$ is supercomplete,

(2) for some, and then any, compactification $K$ of $X$ the following holds:

for each compact $C \subset K \backslash X$ there exists a uniformly continuous function $f$ on $\lambda X * K$ such that $f$ is 1 on $\Delta_{X}$ and $f$ is 0 on $X \times C$,

(3) for some, and then any, compactification $K$ of $X$ the following holds:

for each pair of closed disjoint sets $F_{0}, F_{1}$ in $X \times K$ there exists a uniformly continuous function $f$ on $\lambda X * K$ such that $f$ is 1 on $F_{1}$ and $f$ is 0 on $F_{0}$.

1. Definitions and facts. Following Isbell, we denote the semiuniform product of spaces $X$ and $Y$ by $X * Y$. The underlying set of $X * Y$ coincides with the underlying set of $X \times Y$. The uniformity of $X * Y$ is the weak uniformity induced on $X \times Y$ by all semiuniform mappings onto, mapping $f$ on $X \times Y$ being semiuniform if $\{f(-, y) \mid$ $y \in Y\}$ is an equiuniformly continuous family on $X$ and each $f(x,-)$ is uniformly continuous on $Y$. It is clear that $X * Y$ is homeomorphic to $X \times Y$ and that all semiuniform mappings on $X \times Y$ are uniformly continuous on $X * Y$.

We recall a few facts.

Fact 1 [ $\mathbf{I}_{1}$, III 23]. Every uniform covering of $X * Y$ has a refinement of the form

$$
\left\{U_{\alpha} \times V_{\beta}^{\alpha}\right\}_{\alpha \in A, \beta \in B_{\alpha}}
$$

where $\left\{U_{\alpha}\right\}$ and $\left\{V_{\beta}^{\alpha}\right\}_{\beta \in B_{\alpha}}$ are uniform coverings of $X$ and $Y$, respectively.

Fact 2 [ $\mathbf{I}_{1}$, VII 29]. If $X$ is fine and $Y$ is compact, then $X * Y$ is fine.

Fact 3. If $X$ has a point-finite basis, then for any $Y$, every covering of the form (s) is uniform on $X * Y$. Proof of this statement is almost the same as the proof of the fact that the first Ginsburg-Isbell derivative of $X$ is a uniformity [ $\mathbf{I}_{1}$, VII 5].

Fact $4\left[\mathbf{H}_{1}\right]$. A space $X$ is uniformly paracompact if and only if for some, and then any, compactification $K$ of $X$ and for any compact $C \subset K \backslash X$ there exists a uniform cover $\left\{V_{a}\right\}$ of $X$ such that for each $V_{a}, \bar{V}_{a}^{K} \cap C=\varnothing$.

We shall also need the easy observation mentioned by A. Hohti $\left[\mathbf{H}_{1}\right]$.

Fact 5 . A uniformly paracompact space has a point-finite basis. We give the proof for the reader's convenience.

Suppose that $W$ is a uniform covering of a uniformly paracompact space $X$. Take an open uniform covering $\mathscr{V}, \mathcal{V}^{*} \mathcal{Q}$. $\mathcal{V}$ has an open refinement $\mathcal{G}$ locally finite relative to $\{\operatorname{St}(W, W) \mid W \in \mathscr{W}\}$, $W$ being a uniform covering of $X$, $\mathscr{W} \prec$. Hence $\{\operatorname{St}(G, \mathscr{\mho}) \mid G \in \mathcal{G}\}$ is the desired uniform refinement of $\mathscr{U}$. 


\section{Proofs of theorems.}

Proof of Theorem 1. Suppose that $K$ is a compactification of $X$, let $X \times C$ and $\Delta_{X}$ be separated by a uniformly continuous function for any compact $C \subset K \times X$. Separating the function provides us a covering $\mathscr{Q}=\left\{U_{\alpha} \times V_{\beta}^{\alpha}\right\}$ (of the form (s)) such that $\operatorname{St}\left(\Delta_{X}, \mathcal{Q}\right) \cap X \times C=\varnothing$. Suppose that $c \in \bar{U}_{\alpha}^{K} \cap C$ for some $\alpha$. Then there exists $\beta$ such that $V_{\beta}^{\alpha}$ is a neighborhood of $c$. Thus $V_{\beta}^{\alpha} \cap U_{\alpha} \neq \varnothing$ and $U_{\alpha} \times V_{\beta}^{\alpha}$ meets both sets $\Delta_{X}$ and $X \times C$ which is impossible. Hence, $\bar{U}_{\alpha} \cap C=\varnothing$ for each $\alpha$. According to Fact $4, X$ is uniformly paracompact.

Let $X$ be uniformly paracompact, $K$ be a compactification of $X, C \subset K \backslash X$ be a compact set. Then there exists a uniform covering $\mathcal{V}=\left\{V_{a}\right\}$ such that $\bar{V}_{a}^{K} \cap c=\varnothing$ for each $a$. We may and shall assume that $\mathscr{V}$ is point-finite. Obviously, it is enough to construct a uniform cover $\mho \delta$ of $X * K$ such that $\operatorname{St}\left(\Delta_{X}\right.$, $\left.\sigma\right) \cap X \times C=\varnothing$. Since $\bar{V}_{a}^{K} \cap c=\varnothing$, we can choose a binary open cover $\left\{U_{a}^{1}, U_{a}^{2}\right\}$ of $K$ such that $V_{a} \subset U_{a}^{1}$ and $V_{a} \cap U_{a}^{2}=\varnothing$. Put

$$
\mathscr{W}=\left\{V_{a} \times U_{a}^{i} \mid V_{a} \in \mathcal{V}, i=1,2\right\}
$$

Proof of Theorem 2. $X * K$ is fine and paracompact, provided $X$ is fine and paracompact. So "only if" is obvious.

Suppose that disjoint closed sets can be separated. Then $X$ is paracompact. Let $\left\{U_{a}\right\}$ be an open cover of $X$ and let $\left\{V_{\alpha}\right\}$ be its closed locally finite refinement. For each $\alpha$, take one $a_{\alpha}$ such that $V_{\alpha} \subset U_{a_{\alpha}}$. Then $\left\{U_{a_{\alpha}}\right\} \prec\left\{U_{a}\right\}$. Then $\Delta_{X}$ and $T$ $=\overline{U\left\{V_{\alpha} \times K-U_{a_{\alpha}}\right\}}$ are closed disjoint sets. Existence of separating functions provides us a cover of the form (s), $\mho=\left\{U_{\delta} \times V_{\gamma}^{\delta}\right\}$, such that $\operatorname{St}\left(\Delta_{X}, \mathcal{Q}\right) \cap T=\varnothing$. Let $z \in U_{\delta} \cap V_{\alpha}$. Suppose $U_{\delta} \not \subset U_{a_{\alpha}}$, i.e. there exists $x \in U_{\delta} \backslash U_{a_{\alpha}}$. Take $V_{x}^{\delta}$ such that $x \in V_{x}^{\delta}$. Hence $U_{\delta} \times V_{\gamma}^{\delta} \cap \Delta_{X} \neq \varnothing \neq U_{\delta} \times V_{\gamma}^{\delta} \cap T$ which is impossible.

We recall that $X$ is supercomplete if its hyperspace $H(X)$ of closed subsets (see $\left.\left[\mathbf{I}_{1}\right]\right)$ is complete, which is equivalent $\left[\mathbf{I}_{2}\right]$ to $\lambda X$, is fine and paracompact.

Proof of Corollary. We know that $(1) \rightarrow(3) \rightarrow(2)$. (2) says that $\lambda X$ is uniformly paracompact, hence $\lambda(\lambda X)=\lambda X$ is fine ( $\lambda$ of a uniformly paracompact space is fine [R]) and paracompact.

The equivalence of (1) and (3) has been proved by A. Hohti $\left[\mathbf{H}_{2}\right]$ by a different technique. In fact, he uses separation in $\lambda(X \times K)$, but $\lambda(X \times K)=\lambda X * K$.

Concluding Remark. We recall that $A \subset X$ is a cozero set in $X$ if there exists a uniformly continuous function $f$ on $X$ such that $A=\{x \mid f(x) \neq 0\}$. A real-valued function is called coz-function if the preimage of every open set is a cozero set. Denote by $m X$ the metric-fine coreflection of $X$ (see $\left[\mathbf{F}_{1}\right]$ for the description of $\left.m X\right)$. In $\left[\mathbf{F}_{\mathbf{3}}\right]$ it is proved that for compact $K$,

$$
m X * K=m(X * K)
$$

Since coz-functions on $X$ coincide with uniformly continuous functions on $m X$, the application of our results to $m X$ yields the following parts of two theorems from [ $\left.\mathbf{F}_{3}\right]$. 
THEOREM 1'. The following conditions are equivalent:

(i) $m X$ is uniformly paracompact,

(ii) for some (and then any) compactification $K$ of $X$ and for any compact $C \subset K \backslash X$ there exists a coz-function $g$ on $X * K$ such that $g \mid \Delta_{X}=1$ and $g \mid X \times C=0$.

THEOREM 2'. The following conditions are equivalent:

(i) $m X$ is fine and paracompact,

(ii) for some (and then any) compactification $K$ of $X$ and for each pair $F_{0}, F_{1}$ of closed disjoint sets there exists a coz-function on $X * K$ such that $g \mid F_{0}=0$ and $g \mid F_{1}=1$.

\section{REFERENCES}

$\left[F_{1}\right]$ Z. Frolik, A note on metric-fine spaces, Proc. Amer. Math. Soc. 46 (1974), 111-119.

$\left[\mathbf{F}_{2}\right] \ldots$ _ Paracompact uniform spaces, Czechoslovak Math. J. (to appear).

$\left[\mathbf{F}_{3}\right] \_$, On two classes of paracompact uniform spaces, Časopis Pěst. Mat. (to appear).

$\left[\mathbf{H}_{1}\right]$ A. Hohti, On uniform paracompactness, Ann. Acad. Sci. Fenn. Ser. A I Math., Dissertationes No. 36,1981 .

$\left[\mathbf{H}_{2}\right] \ldots$, On supercomplete spaces, Proc. Amer. Math. Soc. 87 (1983), 557-560.

[I $\left.{ }_{1}\right]$ J. R. Isbell, Uniform spaces, Math. Surveys, No. 12, Amer. Math. Soc., Providence, R. I., 1964.

[I $\left.\mathbf{I}_{2}\right] \ldots$, Supercomplete spaces, Pacific J. Math. 12 (1962), 287-290.

[R] M. D. Rice, A note on uniform paracompactness, Proc. Amer. Math. Soc. 62 (1977), 359-362.

[T] H. Tamano, On paracompactness, Pacific J. Math. 10 (1960), 1043-1047.

Matematicky Ústav ČSAV, Žitná 25, 11567 Praha 1, CZechoslovakia 